ENCYCLOPEDDIE Encyclopédie berbère

BERBERE

1 | 1984

1 | Abadir - Acridophagie

\title{
Abū Yazīd
}

(Mahlad B. Kaydād al-Nukkarī)

A. Golvin et J. Lanfry

\section{OpenEdition}

Journals

Édition électronique

URL : http://journals.openedition.org/encyclopedieberbere/807

DOI : 10.4000/encyclopedieberbere.807

ISSN : 2262-7197

\section{Éditeur}

Peeters Publishers

\section{Édition imprimée}

Date de publication : 1 novembre 1984

Pagination : $97-102$

ISBN : 2-85744-201-7

ISSN : $1015-7344$

\section{Référence électronique}

A. Golvin et J. Lanfry, «Abū Yazīd », Encyclopédie berbère [En ligne], 1 | 1984, document A36, mis en ligne le 01 décembre 2012, consulté le 05 octobre 2020. URL : http://journals.openedition.org/ encyclopedieberbere/807; DOI : https://doi.org/10.4000/encyclopedieberbere.807

Ce document a été généré automatiquement le 5 octobre 2020.

(c) Tous droits réservés 


\title{
Abū Yazīd
}

\author{
(Mahllad B. Kaydād al-Nukkarī)
}

\section{A. Golvin et J. Lanfry}

\section{Par A. GOLVIN}

1 Chef d'une insurrection hāāiǧite (kharédjite) qui devait, au $x^{e}$ siècle, mettre en péril le califat fāțimide d'Ifrīqiya.

La vie de cet illustre aventurier politique reste assez obscure jusqu'à ce qu'il surgisse dans l'histoire à l'âge de soixante ans. A l'aube de la vieillesse, il semblait d'autant moins qualifié pour fomenter une insurrection et prendre la tête de la rébellion qu'il était chétif, difforme et boiteux.

Né vraisemblablement vers $270 / 883$ au Soudan où son père se rendait fréquemment pour y commercer et retourner ensuite au Jérid, il appartenait à une famille berbère ibādite de la confédération des Zanàta, tribu des Banū Ifran. C'est dans la doctrine hārigite qu'il reçut une instruction suffisante pour qu'il enseigne le Coran aux enfants de Tozeur et peut-être aussi à ceux de Tâhert* .

Depuis le début $\mathrm{du} \mathrm{IV}^{\mathrm{e}} / \mathrm{x}^{\mathrm{e}}$ siècle, le Mag̈rib connaissait un profond bouleversement politique. Le dā’i Abū 'Abd Allāh aidé des berbères șanhāğiens Kutāma de petite Kabylie s'était emparé de Kairouan, mettant un terme à la dynastie des Aglabides. Il était allé ensuite délivrer son maître 'Ubayd Allāh à Siğilmāsa, balayant au passage la dynastie hāriğite des Rustamides de Tāhart. Le triomphe du mouvement fāțimide mettait également un terme à la dynastie des Banū Midrār de Siğilmāsa. Enfin, dès son arrivée à Kairouan-Raqqāda, le Mahdi 'Ubayd Allāh avait expédié une armée vers le Mag̉rib alAqșā. Toute l'Afrique du Nord se trouvait alors apparemment unifiée sous l'autorité des Šì'ites. Les communautés ibāḍides, chassées de Tāhert refluaient vers le Jérid où Abū Yazīd se faisait le propagandiste d'un mouvement anti-š̄i ite. Son enseignement jugé dangereux pour le pouvoir central, il est pourchassé, arrêté vers 316/928, mais il est presque aussitôt remis en liberté. Il va alors chercher refuge dans l'Aurès, chez les Howwāra ; il s'y proclame le Šayh des Vrais Croyants. 
4 A nouveau poursuivi, il part accomplir son pèlerinage et il revient clandestinement au Jérid vers 324/937. Il reprend sa propagande anti-šǐitite dans l'Aurès, aidé de ses quatre fils et de son maître aveugle Abū'Ammār 'Abd al-Ḥamid.

La mosquée de Kairouan (Photo G. Camps).

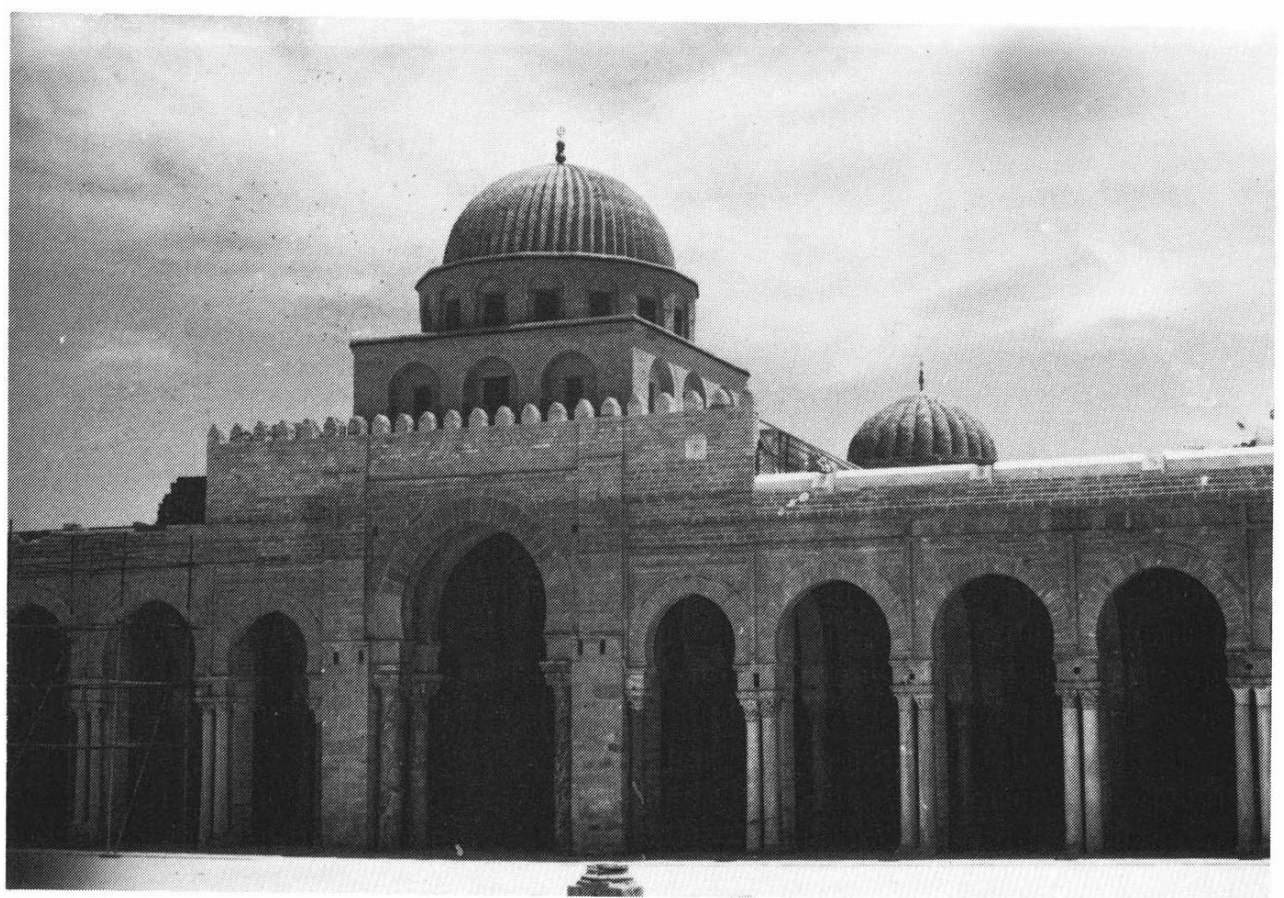

5 Vêtu de bure, vivant dans une simplicité ascétique, il se déplace à dos d'âne d'où son surnom : l'homme à l'âne. Bientôt, il dispose d'une armée et il entre en campagne en $322=943$. La riposte fātimide, sous la forme d'un corps de troupe commandé par 'Ali b.Hamdūn, le seigneur de Msila (un réfugié andalou), s'avère désastreuse, le chef est tué, la panique s'empare de l'armée régulière. Le prestige de Abū Yazīd grandit démesurément. Il est bientôt maître du Zâb et du Jérid. En $333=944$, il fonce sur l'Ifrīqiya, il y trouve un appui précieux auprès des berbères Banū Kamlān précédemment déportés du Mag̉rib central en Ifrīqiya, sa marche triomphale le conduit rapidement à Kairouan où il trouve un accueil quasi inespéré auprès d'une population foncièrement anti-š̄ìite. C'est le 23 șafar 333/15 octobre 944 que le chef rebelle entre triomphalement dans la ville sainte. Malheureusement pour lui, il a la faiblesse de laisser ses hordes piller la capitale, faute politique qu'il paiera cher un peu plus tard. Le Calife fāțimide al-Qā’im réside à Mahdiya ; il a confié le commandement de son armée à un slave : Maysūr. Celui-ci tente de barrer la route aux troupes de Abū Yazīd, il est tué et ses soldats mis en déroute. L'homme à l'âne est aux portes de Mahdiya, il sent la victoire finale à portée de sa main. Alors, il jette sa robe de bure, s'habille de soie, remplace son humble et ridicule monture par un fringant coursier, attitude qui lui aliène bien des sympathies dans le clan de ses plus fidèles alliés. Cependant Mahdiya assiégée connaît les affres de la disette après les terreurs que lui avait inspirées un premier assaut qui avait échoué presque miraculeusement. Le Calife trouve un appui providentiel chez les Șanhāğa du Maġrib central commandés par Zīrī fils de Manàd. Ce dernier réussit à ravitailler la ville et à harceler les arrières de la troupe des assiégeants. Cette arrivée inopinée et la lassitude des combattants de Abū Yazīd 
obligent le rebelle à se replier, il fait marche arrière vers Kairouan où l'accueil de la population est très froid. Il sent qu'il lui faut regagner la confiance des siens et s'imposer à une population hésitante. Il décide alors de revenir à ses habitudes d'austérité et à nouveau il est l'homme à l'âne, rassembleur des énergies. Il va assiéger Sousse tandis qu'un des ses fils, Ayyūb, se bat à Tunis, puis à Béja, mais le général loyaliste al-Ḥasan b.'Alī remporte une série de succès; Ayyūb doit se replier sur le Magrib central. Sur ces entrefaites, al-Qā’im meurt à Mahdiya le 13 sawwāl 334/18 mai 946, on tient secrète la mort du Calife tandis que l'héritier présomptif, al-Manșūr attaque les armées rebelles. Il dégage Sousse, Abū Yazìd se replie sur Kairouan qui refuse de lui ouvrir ses portes. Une bataille furieuse, le 13 muharram $335=14$ août 946 , tourne à l'avantage de al-Mansûr. L'homme à l'âne, serré de près, recule vers l'ouest se défendant avec une farouche énergie qui lui gagne de nouveaux alliés, mais les défaites se succèdent, Maggara doit céder devant les troupes fâtimides, al-Mansùr entre à Msila, Abū Yazīd cherche refuge dans le djebel Salat, près de Bou Saada. Une bataille nouvelle voit l'écrasement des hariğites, Abù Yazīd, blessé, échappe de peu à la mort, il s'évade encore une fois et tente même de reprendre Msila, mais en vain. Il se retranche alors dans la montagne de Maadid; serré de près, il résistera pendant cinq mois à l'assaut des troupes d'Al-Manșūr, il luttera pied à pied jusqu'au sommet du Takerboust où s'élèvera quelques années plus tard la Qal'a des B. Ḥammād. Abandonné de ses plus fidèles alliés, réduit à quelques hommes qui tentent de le protéger, le farouche révolté défend chèrement sa vie. Blessé accidentellement, il est moribond lorsqu'il tombe entre les mains de ses vainqueurs. Il meurt lors de son transport à Msila le 27 muharram $336=20$ août 947.

6 Sur ordre de al-Manșūr, son cadavre est écorché et sa peau bourrée de coton et de paille. Quelque temps après, la sinistre dépouille empaillée, coiffée d'un bonnet de coton et vêtue de vêtements grotesques, est promenée dans les rues de Kairouan, juchée sur un chameau entre deux singes dressés pour souffleter sa pauvre figure, sous les huées d'une foule excitée et apparemment soulagée de ce retour au calme.

7 La dynastie fāțimide était sauvée. On aurait tort cependant de considérer cette rébellion comme un épisode isolé de l'histoire du Mag̈rib et encore davantage de la considérer comme une réaction naturelle des hāriǧites contre le šǐisme. La révolte de l'homme à l'âne peut certes trouver ses raisons dans les conditions sociales nouvelles locales et il est indéniable qu'elle se nourrit d'une opposition religieuse certaine, mais elle s'inscrit avant tout dans un contexte de perpétuelle résistance du monde berbère à toute sujétion d'un pouvoir central établi, ressenti comme étranger. Abū Yazīd a su polariser sur sa personne, au x ${ }^{\mathrm{e}}$ siècle, ce sentiment de rejet comme l'avaient fait avant lui les Jugurtha et les Firmus contre Rome, les Kusayla et la Kahina ainsi que quelques autres chefs ou pseudo prophètes berbères contre l'autorité incarnée par les Emirs arabes. 


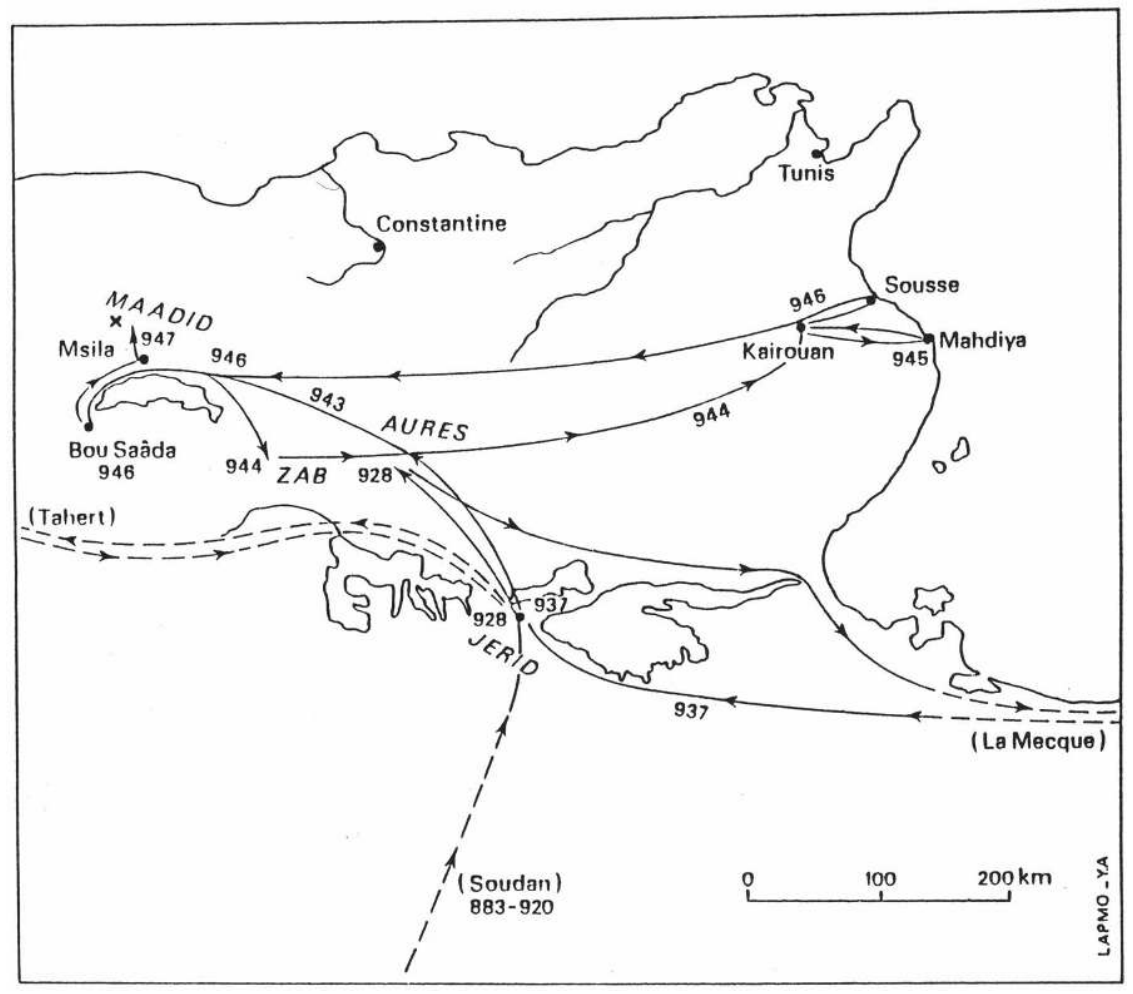

\section{Abū Yazīd - A propos de son surnom : «l'homme à l'âne » (J. LANFRY)}

Le nom complet de ce personnage historique, ce Berbère kharédjite, révolté, que les chroniqueurs et historiens arabes, et après eux les historiens européens, désignent moins par son vrai nom que par son sobriquet, «l'homme à l'âne ", s'énonce ainsi, à la manière classique : Abū Yazīd Mah̆lad ibn Kaydād. Abū Yazīd est le surnom, la kunya. Le nom personnel est Mahllad. Le père de Mahlad était originaire du pays de Qastīliya (Ğarīd, région des Chotts du Sud tunisien) et plus précisément de la ville de Tawzer (Tozeur) où il résidait. Certains ont mentionné aussi comme lieu de séjour et peut-être d'origine de ce Kaydād, un village du Ğebel Nefūsa, Taqyus. Il voyageait avec des caravanes qui faisaient trafic commercial au Bilād al-Sūdān. Il prit femme dans l'oasis saharienne de Tadmekket (Tademket qui serait aujourd'hui : Es-Souk, au Mali, dans l'Adrar des Ifoyas). Sa femme, une esclave de la tribu berbère des Huwwāra, donna naissance à Mahllad, notre héros.

L'enfant, premier-né de Mahlad, reçut de son père le nom de Yazīd: d'où le surnom porté par le père, qui est la kunya: en arabe : Abū Yazīd. La kunya fut souvent une manière de distinction, de titre de respect auquel on tenait beaucoup. On sait que Abū Yazīd fut un adepte et un apôtre de kl'ibadisme nakkarite. Il étudia et enseigna à Tahert, avant de revenir, déjà âgé, au Ğarīd. Les historiens rapportent que peu après son retour au pays de son père, ayant prêché la doctrine kharedjite, il rencontra de la part des autorités chiites une vive résistance à son action, qui le conduisit à la révolte. Il soulève alors les populations berbères entre le Ğarīd et l'Awras (332/943). Abū Yazīd prit Tébessa et Marmadjanna. C'est dans cette localité qu'un partisan lui offrit un âne 
gris qu'il prit comme monture habituelle. Et dès lors, rapporte Ibn Khaldoun après d'autres chroniqueurs, il fut connu sous le nom de «l'homme à l'âne », en arabe : șahib al-himār (Ibn Khaldoun, Histoire, trad. de Slane, t. II, p. 531). On pourrait s'en tenir là, enregistrer cette information, sans plus, comme l'ont fait par la suite les historiens européens qui se sont intéressés à l'épisode dramatique de la révolte au Maghreb Oriental de "l'homme à l'âne " (qui dura de 943 à $947 \mathrm{~J}$.-C.) Ce sobriquet garde le souvenir de cette assez banale histoire de la monture de Mahlad.

C'est peu. Un berbérophone de ces régions, à mon avis, goûtait un peu plus de sel, si je ne me trompe, à prononcer le nom du meneur ibadite révolté, sel que ne pouvait sentir un arabophone. Voici le fait.

11 Le nom (kunya) d'Abū Yazīd est d'origine arabe, sans aucun doute. Il se trouve que ce nom a une consonance berbère. Quand on dit Abu Yazid et qu'on est Berbère des régions mentionnées plus haut, l'idée peut venir très spontanément d'exprimer autre chose que "père de Yazid». Car, à très peu près, on dit, en berbère cette fois, "l'homme à l'âne ». Il y a donc un calembour, un jeu de mots possible, qui n'était possible qu'aux Berbères de ces régions et qui restait inintelligible aux arabophones. Les faits linguistiques sont simples:

12 a) L'élément bu est berbère, on le sait, et fréquemment utilisé comme composant de noms et de surnoms : il se confond de fait avec l'arabe abu dans sa forme dialectale bu. En berbère, bu signifie (en ar. dialectal aussi) possesseur de, l'homme qui, l'homme à, celui de. Il constitue le premier élément d'un grand nombre de complexes $(b u+$ substantif) où il marque une relation d'appartenance, une propriété, stable ou non, caractéristique, définie par le substantif déterminant qui suit.

b) Les dialectes berbères orientaux et Kabyle désignent l'âne par des noms dont la racine fondamentale commune est Z D ou Y Z D (en touareg du Hoggar Y H D ). Les réalisations sont diverses selon les dialectes et les parlers; par ex., chez les Touareg: eyhị, ajiḍ, ijiḍ ; à Ghadamès : azị̣ (J. Lanfry, Ghadamès II, Glossaire, 1973).

Ces indications lexicographiques suffisent à notre propos : un Berbère saharien pouvait passer aisément, au prix d'une emphase accordée à la dernière radicale dentale sonore $\mathrm{d}$, du nom de Yazīd à celui de sa monture. En prononçant : a bu-y-azị, on disait : ô l'homme à l'âne !

Bien sûr, personne, n'aurait eu ni raison ni envie de jouer sur les mots si Mahlad n'avait reçu en cadeau un âne et s'il n'en avait fait, pendant un temps au moins, sa monture ordinaire, comme un signe de son choix doctrinal et spirituel: pauvreté et renoncement qui lui valurent au début une part de sa popularité.

Les Arabes n'ont retenu en tout cas que l'histoire de l'âne sans faire le rapprochement avec la kunya, le surnom par lequel on désignait son propriétaire. Et en somme, le sobriquet arabe, șăhib al-himār, ne serait venu qu'après coup, comme une simple traduction du calembour que permettait la langue berbère. C'est un fait qu'aucun historien arabe ne mentionne l'explication qu'on propose ici. Nous avons admis facilement, trop à mon sens, l'explication du sobriquet en langue arabe, sans prendre garde que le peuple, les tribus, les contrées que fréquentait Abū Yazīd, parmi les pauvres gens des campagnes et des montagnes parlaient berbère et n'étaient guère bilingues à cette époque. Le contexte berbérophone était alors, entre Ǧarīd et Awrās, et même jusqu'à Kairouan et Béja, infiniment plus dense que la situation actuelle ne le laisse imaginer. Si on a surnommé Abū Yazīd, l'homme à l'âne, c'est en berbère qu'on 
l'a fait d'abord. Or, il n'y avait pas à inventer ni ajouter : c'était déjà presque son nom, en berbère.

En lisant le texte arabe d'Ibn Hammād sur l'épisode qui nous occupe, on peut se demander si cet auteur, qui fut sans doute une des sources d'Ibn Khaldoun, n'avait pas eu lui-même la clef de notre modeste problème. Il relève ces paroles prêtées au Mahdi 'Ubayd Allah, qui aurait eu une intuition de l'avenir et des tentatives de conquête de sa capitale toute neuve, Mahdiya : "C'est jusqu'ici qu'arrivera l'homme à l'âne, șāhib alhịmār yazni Abū Yazīd, l'homme à l'âne, qui veut dire Abū Yazīd, qui désigne A. Yazīd ». L'anecdote est racontée par Ibn Khaldoun dans la Muqaddima (Voir traduction V. Monteil : Discours sur l'Histoire Universelle, Beyrouth 1968, t. II, p.689). Il y a une possibilité de lire ainsi, en y trouvant une discrète indication de ce que nous avons expliqué. Le texte reste ambigu, il faut le reconnaître. Le recours à la langue berbère, normal dans ce cas, est certainement plus parlant.

\section{BIBLIOGRAPHIE}

Auteurs arabes :

AL-MĀLIKI, Riyād al-Nufūs, Le Caire, 1951 (R.H. Idris, Contribution à l'histoire de l'Ifrìqiya, Tunis, 1935-1936).

AL-DARĞINĪ, Kitāb al-Tabaqāt, manuscrit (v. Le Tourneau).

'IMĀD AL-DĪN IDRĪs, 'Uyūn al-Ahbār (v. S.M. Stem).

ABŪ ZAKARIYYĀ, Chronique (Masqueray) Alger, 1879.

IBN ‘IDĀRĪ, Bayān I, Fagnan, 1901.

IBN KHALDOUN, Berbères, de Slane, III, 201.

IBN ḤAMMĀD Histoire des rois 'Obaidides, Vonderheyden, Alger, 1927.

AL-BAKRĪ, Kitāb al-Masālik, de Slane, Alger, 1911.

AL-TIǦĀNī, Rihla, Abdul-Wahab, Tunis, 1958.

Auteurs contemporains :

STERN S.M. art. Abū Yazìd, Encyclopédie de l'Islam, I, 167-168.

MARÇAIS G. La Berbérie musulmane, Paris, 1951.

LE TOURNEAU R. La révolte d'Abû Yazîd au Xe siècle, Les Cahiers de Tunisie, n. ${ }^{\circ}$ 2, 2e trim. 1953.

IDRIS R.H. La Berbérie Orientale sous les Zīrides, X-XIIe siècle, Paris, 1962, I, 17 à 24.

INDEX

Mots-clés : Algérie, Biographie, Moyen Âge, Onomastique 